\title{
D-Test for Detection of Antimicrobial Susceptibility in Methicillin Resistant Staphylococcus Aureus (MRSA)
}

\author{
Flora Grace M \\ (Ph.D.) Research Scholar Department of Zoology, Madras Christian College (Autonomous), Tambaram, Tamil \\ Nadu, India,
}

\begin{abstract}
Clindamycin is an alternative choice for mild to moderate MRSA infections especially in penicillin-allergic patients. Clindamycin has been used to treat serious infections caused by susceptible Staphylococcus aureus strains in children for more than 30 years. It remains effective for many infections caused by community-acquired methicillin-resistant Staphylococcus aureus (CA-MRSA). The clinical implications of a positive D-test begin with an understanding of cross resistance for 3 antibiotic families that share a common binding site-macrolides (e.g., Erythromycin) Lincosamide (e.g.; Clindamycin), and group B streptogrammins. A positive D-test indicates the presence of MLSBi genotype. However, sub inhibitory concentration of Erythromycin is a common inducer of Inducible Clindamycin resistance (ICR). When Erythromycin diffuses, it induces, resistance to Clindamycin and results in flattening of the Clindamycin zone of inhibition just next to the Erythromycin disk, making a D shape, so this method is called D-test. Susceptibility testing was performed according to BSAC (British society for antimicrobial chemotherapy) on isolates of Staphylococcus aureus by using the method of disk diffusion. All MRSA isolates processed were found to have positive D Test. Staphylococcus aureus is one of the most common human pathogens with ability to cause wide range of infections.
\end{abstract}

Keywords: Erythromycin, Clindamycin, Disc Diffusion, BSAC, $M L S B c, M L S B i, I C R$

\section{Introduction}

Staphylococcus aureus is one of the most common human pathogens with ability to cause wide range of infections. Although healthy children have a small risk for serious infections, but they could be common carriers of this organism and many clinical infections may develop in nasal carrier individuals. The incidence of invasive infections has been raising with emergence of community acquired (CA) and Hospital Acquired (HA) Methicillin Resistant Staphylococcus aureus (MRSA). Clindamycin is an alternative choice for mild to moderate MRSA infections especially in Penicillin allergic patients. Clindamycin has been used to treat serious infections caused by susceptible Staphylococcus aureus strains in children for more than 30 years. It remains effective for many infections caused by Community-Acquired Methicillin Resistant Staphylococcus aureus (CA -MRSA).

Clindamycin also is useful for selected infections caused by pneumococci, group A streptococci, and a number of other microbes. Absorption after oral administration is nearly complete, yielding serum concentrations that approximate those from intravenous administration. Clindamycin resistance is common among health care-associated MRSA strains. Most CA-MRSA remain susceptible to date, but resistance rates vary by region. Macrolide - inducible resistance to clindamycin was first recognized in the laboratory in the early 1960s.

The clinical implications of a positive D-test begin with an understanding of cross resistance for 3 antibiotic families that share a common binding site-macrolides (e.g., Erythromycin) Lincosamide (e.g.; Clindamycin), and group B streptogrammins. A positive D-test indicates the presence of MLSBi genotype. This means that it is possible, but far from certain, that a subpopulation of microbes resistant to Clindamycin may emerge and lead to clinical failure. Infections caused by Staphylococcus aureus strains that carry the MLSBi genotype often respond to Clindamycin therapy without relapse resistant subpopulations either do not develop or are eliminated by host responses before progression of infection can occur.

However, sub inhibitory concentration of Erythromycin is a common inducer of Inducible Clindamycin resistance (ICR). Our study estimates the frequency of D-test among Staphylococcus aureus isolates. The main objective is to determine the D test in MRSA (Methicillin Resistant Staphylococcus aureus)

\section{A. Methods:}

\section{Performance Evaluation Of D-Test}

It was a cross-sectional study conducted from 2010 to 2011 for about 100 samples which are screened for D test by Disc diffusion method in a tertiary care Hospital in south India. The D test, based on disk diffusion susceptibility testing is recommended to determine if the MLSBi genotype is present. In the D - test disks 
containing Erythromycin $(15 \mu \mathrm{g})$ and Clindamycin $(2 \mu \mathrm{g})$ are placed 15 to $20 \mathrm{~mm}$ apart on an agar plate that has been inoculated with the clinical isolate. A Clindamycin -susceptibility was observed.

\section{B. Purpose:}

1. To use $\mathrm{D}$ - test routinely in all microbiologic laboratories;

2. Not to apply Clindamycin, in patients with infections caused by inducible resistant Staphylococcus aureus.

3. To avoid switch therapy from erythromycin to clindamycin.

\section{Source of sample collection:}

100 isolates of MRSA are obtained from various sources like blood culture, pus, pus swabs, and surgical site infections.

\section{Methodology:}

To detect inducible Clindamycin resistance, there is a specific disc diffusion method that shows resistance, is induced by Erythromycin. In this method an Erythromycin disk is placed next to Clindamycin disk. When Erythromycin diffuses, induces, resistance to Clindamycin and results in flattening of the Clindamycin zone of inhibition just next to the Erythromycin disk, making a D shape, so this method is called D-test. Our study estimates the frequency of D-test among Staphylococcus aureus. Susceptibility testing was performed according to BSAC (British society for antimicrobial chemotherapy) on isolates of Staphylococcus aureus by using the method of disk diffusion. Muller- Hinton agar and Oxacillin, Erythromycin, Clindamycin, and Vancomycin disks were used to determine susceptibility of isolates. D-test was also performed to asses ICR in Erythromycin resistant Clindamycin - susceptible (ER-R/CL-S) isolates. This test was performed by placing an Erythromycin disk in a distance of $15-20 \mathrm{~mm}$ of Clindamycin disk. Staphylococcus aureus is a significant risk factor for serious infections and in some situations elimination of Staphylococcus aureus may reduce rate of subsequent invasive infections.

\section{Result}

All MRSA isolates processed were found to have positive D Test. Staphylococcus aureus is one of the most common human pathogens with ability to cause wide range of infections.

\section{Discussion}

The incidence of invasive infections has been raising with emergence of community acquired (CA) and Hospital-Acquired (HA) Methicillin Resistant Staphylococcus aureus (MRSA). However, sub inhibitory concentration of Erythromycin is a common inducer of inducible Clindamycin resistance (ICR).

TABLE I

Analysis of D-Test

\begin{tabular}{|c|c|c|c|c|c|c|c|c|}
\hline \multicolumn{9}{|c|}{ MIC and Zone diameter breakpoints of Erythromycin and Clindamycin ( D test ) } \\
\hline & \multicolumn{3}{|c|}{$\begin{array}{c}\text { MIC Breakpoint } \\
(\mathrm{mg} / \mathrm{L})\end{array}$} & & \multicolumn{2}{|c|}{$\begin{array}{c}\text { Interpretation of } \\
\text { Zone } \\
\text { diameters }(\mathrm{mm})\end{array}$} & \multirow[b]{2}{*}{$\mathrm{S}>=$} & \multirow[b]{2}{*}{ Comment } \\
\hline Antibiotic & $\mathrm{R}>$ & I & $\mathrm{S}<=$ & $\begin{array}{c}\text { Disc } \\
\text { Content }(\mu \mathrm{g})\end{array}$ & $\mathrm{R}<=$ & I & & \\
\hline Clindamycin & 0.5 & 0.5 & 0.25 & 2 & 22 & $23-25$ & 26 & $\begin{array}{l}\text { Organisms that appear resistant to } \\
\text { Erythromycin, but susceptible to } \\
\text { Clindamycin should be checked for the } \\
\text { presence of inducible resistance If } \\
\text { positive, report as resistant to } \\
\text { clindamycin or report as susceptible } \\
\text { with a warning that clinical failure } \\
\text { during treatment with clindamycin } \\
\text { may occur by selection of } \\
\text { constitutively resistant mutants and the } \\
\text { use of clindamycin best avoided in } \\
\text { severe infection }\end{array}$ \\
\hline Erythromycin & 2 & 2 & 1 & 5 & 16 & $17-19$ & 20 & \\
\hline
\end{tabular}

For serious infections such as sepsis, pneumonia, or other invasive Staphylococcus aureus infections, even the small risk of emergence of resistance to Clindamycin as indicated by a positive D-test should lead to avoidance of use of Clindamycin. The lack of a zone of inhibition around the Erythromycin disc indicates bacterial resistance to macrolides. The large clear zone of inhibition around the Clindamycin disc indicates 
sensitivity to Clindamycin depicts a positive D-test. Diffusion of Erythromycin from the disc towards the Clindamycin disc does not kill bacteria due to Staphylococcus aureus resistance to macrolides. However, in this case the bacterial isolate contains a strain of Staphylococcus aureus with an erythromycin-inducible methylase (MLSBi) that is encoded by a plasmid-borne gene (erm), that both Erythromycin and Clindamycin bind to, making both antibiotics ineffective (inducing resistance). As a result, as Erythromycin diffuses outward into Zone, bacterial resistance to Clindamycin is induced prior to its diffusion from the neighboring disk. In contrast, growth inhibiting concentrations of clindamycin reach zone before Erythromycin can arrive to induce resistance (due to the shorter distance for diffusion), resulting in inhibited growth. The inhibition of bacterial growth produces D shape surrounding the Clindamycin disk, which is considered a D-test.

Macrolide-resistant isolates of Staphylococcus aureus may express constitutive or inducible resistance to clindamycin or may be resistant only to macrolides. Inducible Clindamycin resistance can be detected using a disk diffusion test with Clindamycin and Erythromycin disks placed in close proximity. Flattening of the Clindamycin zone adjacent to the Erythromycin disk (referred to as the D-Zone) indicates inducible Clindamycin resistance. Following incubation, organisms that do not show flattening of the clindamycin zone adjacent to the erythromycin disk should be reported as tested (i.e, susceptible or intermediate to Clindamycin).

While Clindamycin may still be effective in some patients with this phenotype, the working assumption is that the isolate is "presumed" to be resistant based upon detection of inducible Clindamycin resistance. For serious infections such as sepsis, pneumonia, or other invasive Staphylococcus aureus infections, even the small risk of emergence of resistance to Clindamycin as indicated by a positive D-test should lead to avoidance of use of Clindamycin.

\section{A. Interpreting Clindamycin Susceptibility:}

- Initial susceptibility report....

- Erythro-R \& Clinda-R $\rightarrow$ Resistant to clindamycin

- Erythro-S \& Clinda-S $\rightarrow$ Susceptible to clindamycin

- Erythro-R but Clinda-S $\rightarrow$ Need D test to confirm Clinda resistance

- D test

- NEGATIVE = Clindamycin susceptible (efflux mechanism of erythro resistance)

- $\quad$ POSITIVE $=$ Inducible clindamycin resistance $\left(\mathrm{iMLS}_{\mathrm{B}}\right)$

- Inducible clindamycin resistance

- Avoid clindamycin for serious infections if iMLS present

- Clindamycin treatment: Inducible strain-> selection of mutant with constitutive production -> treatment failure

- Occurs without macrolide exposure in patient

- Less certain relevance in less serious infections

- Frequency varies by region and over time

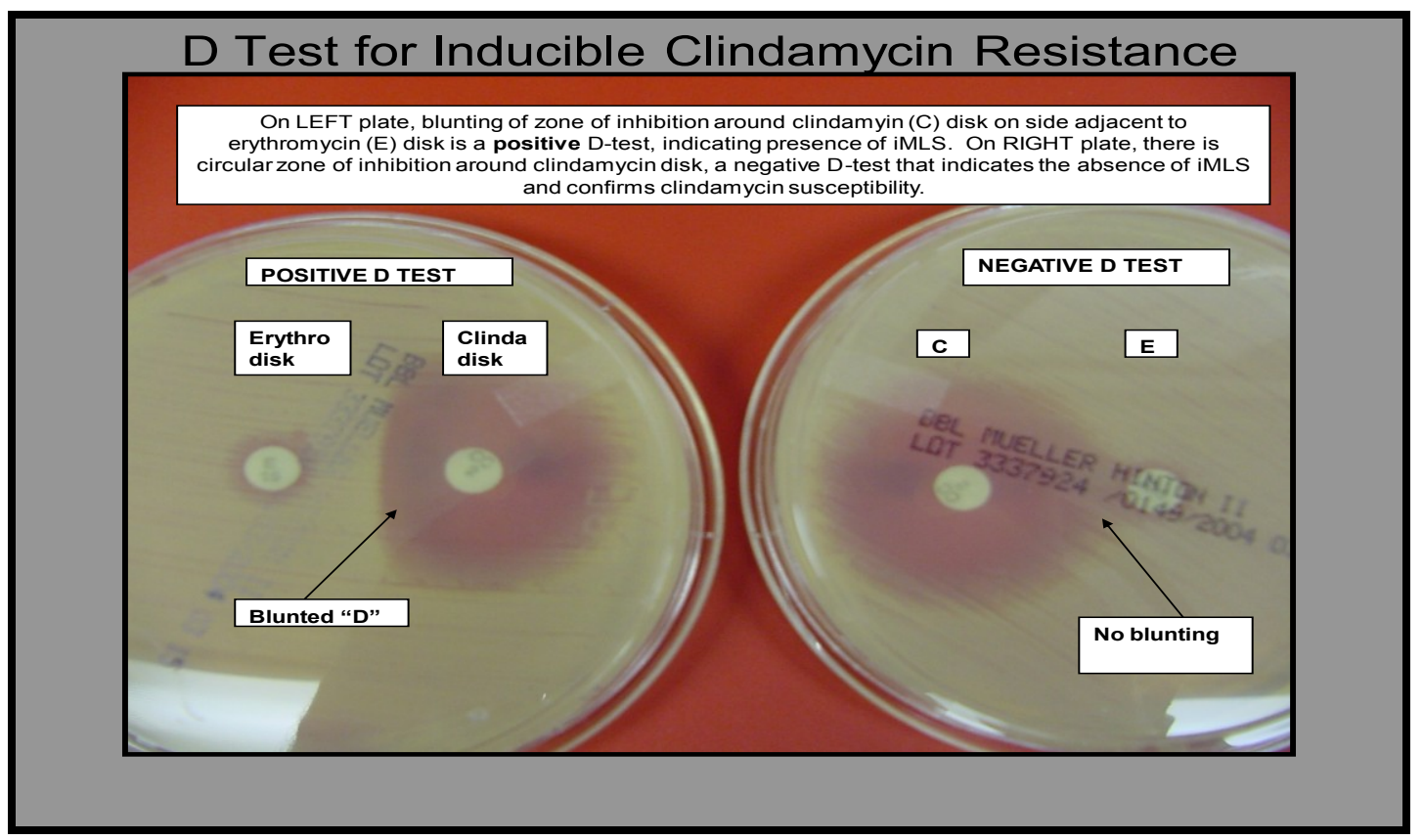

Fig. 1. D-Test for Inducible Clindamycin Resistance 


\section{Conclusion}

The samples processed with BSAC guidelines for D test of MRSA were found to be sensitive to Clindamycin.

\section{References}

[1]. Andrews, J.M. Determination of minimum inhibitory concentrations. Journal of Antimicrobial Chemotherapy, Suppl S1 to Volume 48 July 2001.

[2]. Andrews, J. M., Jevons, G., Brenwald, N. and Fraise, A. for the BSAC Working Party on Sensitivity Testing. Susceptibility testing Pasteurella multocida by BSAC standardized methodology. Journal of Antimicrobial Chemotherapy

[3]. Feibelcorn KR, Crawford SA, McElmeel ML, et al. Practical disk diffusion method for detection of inducible clindamycin resistance in Staphylococcus aureus and coagulase-negative staphylococci. J Clin Microbial. 2003;41(10):4740-4.

[5]. Frank AL, Marcinak JF, Mangat PD, et al. Clindamycin treatment of MRSA infections in children. Pediatr Infect Dis J. 2002; 21(6): $530-4$.

[6]. Roberts MC, Sutcliffe J, Courvalin P, et al. Nomenclature for acrolidelincosamide-streptogramin B resistance determinants. Antimicrobe Agents Chemother. 1999;43(12):2823-30.

[6]. Corea E, De Silva T, Perera J. MRSA: prevalence, incidence and risk factors associated with colonization in Sri Lanka. J Hosp Infect. 2003;55(2): 145-8.

[7]. Fiebelkorn KR, Crawford SA, McElmeel ML, Jorgensen JH (2003): Practical disk diffusion method for detection of inducible Clindamycin resistance in Staphylococcus aureus

\section{AUTHOR PROFILE:}

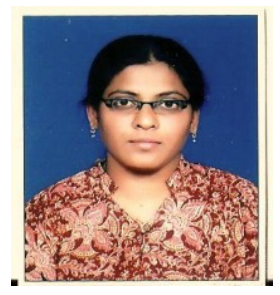

M Flora Grace pursuing her Ph. D from Madras Christian College affiliated to Madras University. Completed post graduation in general microbiology in the year 2006 from Acharya Nagarjuna University. Area of interest is in Medical Microbiology related to Hospital Acquired Infections (HAI's). 